\title{
Análisis de terceros molares y sus estructuras anatómicas adyacentes mediante
} CBCT: meta-análisis

\author{
Analysis of third molars and their adjacent anatomic structures by means of CBCT: meta-analysis \\ Análise de molares de terceiros e suas estruturas anatômicas adjacentes por meios de TCFC: meta- \\ análise
}

Recibido: 23/08/2021 | Revisado: 26/08/2021 | Acepto: 27/08/2021 | Publicado: 29/08/2021

\author{
Jéssica Belén Bermeo Domínguez \\ ORCID: https://orcid.org/0000-0002-0273-9664 \\ Universidad de Cuenca, Ecuador \\ Email: belubd@ hotmail.com \\ Pablo Mateo Morales González \\ ORCID: https://orcid.org/0000-0001-5166-8180 \\ Universidad de Cuenca, Ecuador \\ Email: mateo_mg019@hotmail.com \\ Manuel Estuardo Bravo Calderón \\ ORCID: https://orcid.org/0000-0003-2968-1519 \\ Universidad de Cuenca, Ecuador \\ Email: estuardo.bravo@ucuenca.edu.ec
}

\begin{abstract}
Resumen
El siguiente meta-análisis tiene por objetivo evaluar la posición de los terceros molares mandibulares y sus estructuras anatómicas cercanas (conducto dentario inferior, nervio dentario inferior, cortical lingual, segundo molar inferior). Se realizó mediante un filtro que permite la clasificación y evaluación de diversos artículos científicos, aplicando una búsqueda avanzada a través de bases digitales como PubMed, Cochrane, Science Direct y Wiley, los artículos seleccionados debían ser publicados entre los años 2017 - 2021. Además, detallamos las diferentes clasificaciones utilizadas para evaluar un tercer molar mandibular impactado, que según Winter la posición más prevalente es la mesioangular y según Pell y Gregory existe mayor prevalencia de la Clase 2-B; de igual manera detallamos clasificaciones recientes para molares impactados como son "Liqun Gu" y "Ogüz Boraham". Estos hallazgos ilustran la importancia de ubicar estructuras cercanas al tercer molar mandibular, conocer diferentes clasificaciones para determinar la posición de un tercer molar impactado y la ventaja que presenta el CBCT en la planificación quirúrgica, evitando así posibles iatrogenias en la práctica clínica.

Palabras Clave: Tercer molar; Clasificación de Pell y Gregory; Clasificación de winter; Cone beam; Tomografía Computarizada de haz Cónico - CBCT.
\end{abstract}

\begin{abstract}
The following meta-analysis aims to evaluate the position of mandibular third molars and their nearby anatomical structures (lower dental canal, lower dental nerve, lingual cortex, lower second molar). It was performed using a filter that allows the classification and evaluation of various scientific articles, applying an advanced search through digital databases such as Pubmed, Cochrane, Science Direct and Wiley, the selected articles should be published between 2017-2021. In addition, we detail the different classifications used to evaluate an impacted mandibular third molar, which according to Winter the most prevalent position is the mesioangular and according to Pell and Gregory there is a higher prevalence of Class 2-B; we also detail recent classifications for impacted molars such as "Liqun Gu" and "Ogüz Boraham". These findings illustrate the importance of locating structures close to the mandibular third molar, knowing different classifications to determine the position of an impacted third molar and the advantage of CBCT in surgical planning, thus avoiding possible iatrogenesis in clinical practice.
\end{abstract}

Keywords: Third molar; Pell and Gregory classification; Winter classification; Cone beam; Cone Beam Computed Tomography - CBCT.

\section{Resumo}

A meta-análise a seguir visa avaliar a posição dos terceiros molares inferiores e de suas estruturas anatômicas próximas (canal dentário inferior, nervo dentário inferior, cortical lingual, segundo molar inferior). Foi realizado por meio de um filtro que permite a classificação e avaliação de diversos artigos científicos, aplicando uma busca avançada em bases de dados digitais como PubMed, Cochrane, Science Direct e Wiley, os artigos selecionados 
deveriam ser publicados entre os anos de 2017-2021. Além disso, detalhamos as diferentes classificações utilizadas para avaliar um terceiro molar inferior impactado, que segundo Winter a posição mais prevalente é a mesioangular e segundo Pell e Gregory há uma maior prevalência da Classe 2-B; da mesma forma, detalhamos classificações recentes para molares impactados, como "Liqun Gu" e "Ogüz Boraham". Esses achados ilustram a importância de se localizar estruturas próximas ao terceiro molar inferior, conhecer diferentes classificações para determinar a posição de um terceiro molar retido e a vantagem da TCFC no planejamento cirúrgico, evitando assim possível iatrogênese na prática clínica.

Palavras-chave: Terceiro molar; Classificação de Pell e Gregory; Classificação de winter; Cone beam; Tomografia Computadadorizada de Feixe Cônico - TCFC.

\section{Introducción}

La literatura reporta que los terceros molares mandibulares (3MM) son los dientes con mayor variabilidad anatómica, impactación y anatomía topográfica impredecible (Rivera-Herrera et al., 2020).

Para determinar la ubicación de estos molares en relación con las estructuras anatómicas adyacentes se ha utilizado la radiografía panorámica (RP), cabe recalcar que este método de obtención de imágenes en dos dimensiones presenta superposición de estructuras anatómicas que influyen de manera directa en la toma de decisiones clínicas, por lo que se ha requerido otro tipo de análisis como la tomografía computarizada de haz cónico (CBCT) que permite la visualización sin superposición de estructuras adyacentes (de Oliveira Moreira et al., 2020).

En nuestro medio es importante conocer la clasificación en cuanto a la ubicación y posición de los terceros molares teniendo en cuenta que las clasificaciones más utilizadas son de Pell y Gregory y de Winter.

Este estudio tiene el objetivo de determinar la relación entre la posición de los terceros molares y sus estructuras adyacentes mediante una imagen $3 \mathrm{D}$.

\section{Materiales y Métodos}

\subsection{Problema}

Para la presente investigación nos basamos en los criterios PRISMA, y la principal pregunta de investigación se formuló siguiendo el formato PICO (Tabla 1).

Tabla 1. Formato PICO.

\begin{tabular}{|c|l|}
\hline P & $\begin{array}{l}\text { Limitación de una imagen radiográfica } 2 \mathrm{D} \text { en relación de un tercer molar inferior } \\
\text { con sus estructuras adyacentes. }\end{array}$ \\
\hline I & $\begin{array}{l}\text { Análisis en recostrucción 3D para definir la relación anatómica de los terceros } \\
\text { molares inferiores. }\end{array}$ \\
\hline C & $\begin{array}{l}\text { Diferencia entre la relación dental con sus estructuras anatómicas adyacentes } \\
\text { observadas en una radiografia panorámica. }\end{array}$ \\
\hline O & $\begin{array}{l}\text { Determinar la posición de los terceros molares inferiores en relación con sus } \\
\text { estructuras anatómicas entre estudios } 2 \mathrm{D} \text { y } 3 \mathrm{D} .\end{array}$ \\
\hline
\end{tabular}

Fuente: Autores.

El objetivo general es determinar la relación entre la posición de los terceros molares y sus estructuras adyacentes mediante una imagen $3 \mathrm{D}$. 


\subsection{Criterios de inclusión}

- Revisiones sistemáticas, meta-análisis, estudios comparativos y estudios de casos.

- Publicaciones recopiladas en las siguientes bases de datos: PubMed, Cochrane, Science Direct y Wiley.

- Artículos en inglés y español publicados durante los últimos 5 años.

- Pacientes de género masculino y femenino.

- Pacientes con presencia de al menos un tercer molar inferior.

\subsection{Criterios de exclusión}

- Artículos que no se encuentren dentro del periodo de tiempo establecido.

- Estudios realizados en animales.

- Pacientes bajo tratamiento farmacológico de AINES y/o terapia antirresortiva.

- Pacientes con patologías asociadas a la presencia de un tercer molar.

- Terceros molares con presencia de dolor.

- Lesión de nervio dentario inferior postextracción.

\subsection{Selección de estudios}

La estrategia de búsqueda aplicada en las bases de datos electrónicas PubMed, Cochrane, Science Direct y Wiley (a partir del año 2017) se muestra en la Tabla 2.

Tabla 2. Formato PRISMA.

\begin{tabular}{lll}
\hline Base de Datos & \multicolumn{1}{c}{ Palabras Clave } & Limitaciones \\
\hline \multirow{3}{*}{ PubMed } & - Third molar, Pell and Gregory classification. & \\
& - Third molar, Winter. & - Idioma: inglés. \\
& - Third molar, Winter classification, cone bean. & - Años: 2017-2021. \\
& - Third molar, Pell and Gregory, CBCT. & \\
\hline \multirow{3}{*}{ Science Direct } & - Third molar, Winter classification. & \\
& - Third molar, Winter. & - Idioma: inglés. \\
& - Third molar, Winter classification, cone bean. & - Años: 2017-2021. \\
& - Third molar, Pell and Gregory classification. & \\
\hline \multirow{2}{*}{ Cochrane } & - Third molar, Pell and Gregory classification, CBCT. & \\
\hline \multirow{2}{*}{ Wiley } & Third molar, CBCT. & - Idioma: inglés. \\
& & - Años: 2017-2021. \\
\hline
\end{tabular}

Fuente: Autores.

\subsection{Métodos de revisión}

Una vez aplicadas las palabras claves en las diferentes bases digitales, se procedió a establecer filtros de búsqueda para limitar los estudios según el año de publicación y el tipo de estudio. Los artículos obtenidos fueron evaluados según su título, año de publicación, resumen y resultados.

$\mathrm{Al}$ analizar los diferentes artículos de manera independiente se incluyeron aquellos que concordaban con el objetivo de esta revisión, dirigida a determinar la relación entre la posición de los terceros molares y sus estructuras adyacentes mediante una imagen 3D.

La selección de estudios incluidos se detalla en el diagrama de flujo PRISMA (Figura 1). 
Research, Society and Development, v. 10, n.11, e226101119723, 2021

(CC BY 4.0) | ISSN 2525-3409 | DOI: http://dx.doi.org/10.33448/rsd-v10i11.19723

Figura 1. Diagrama PRISMA de selección de artículos.
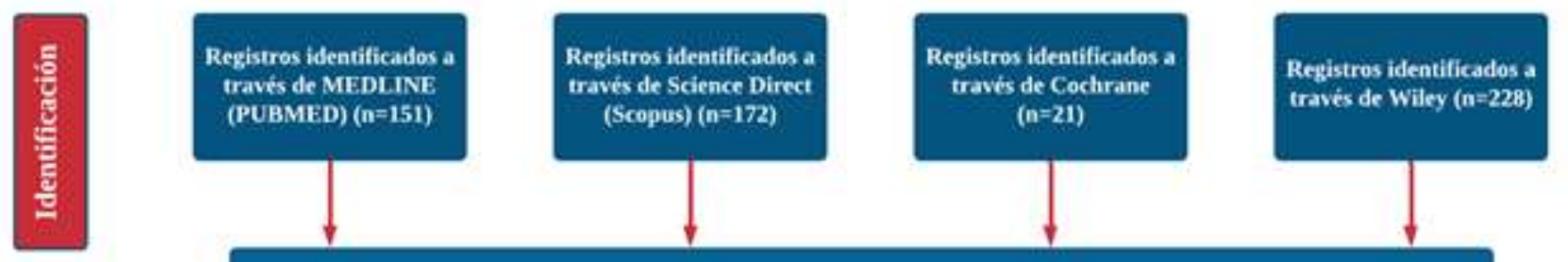

Registros después de eliminar los duplicados $(n=488)$
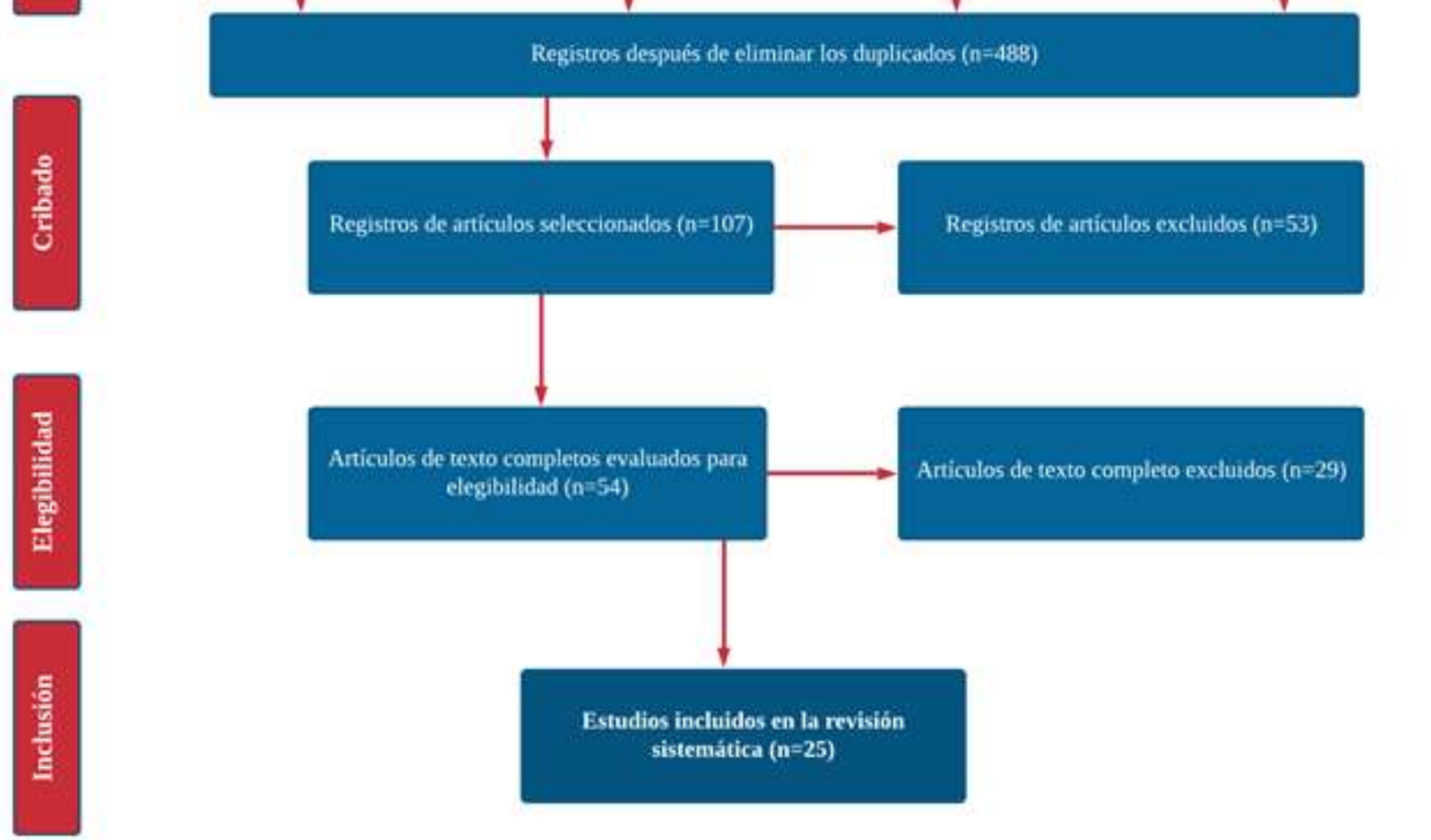

Fuente: Autores.

\subsection{Consideraciones incluidas en esta revisión}

\subsubsection{Clasificación de Pell y Gregory}

Esta clasificación analiza la posición dental en dos criterios (Tabla 3):

Tabla 3. Clasificación de Pell y Gregory.

\begin{tabular}{|c|c|c|c|}
\hline \multicolumn{2}{|c|}{$\begin{array}{l}\text { Distancia entre borde anterior de la rama ascendente - cara distal de } \\
\qquad \text { segunde molar inferior. }\end{array}$} & \multicolumn{2}{|r|}{ Profundidad del diente en relacion al plano oclusal. } \\
\hline Clase 1 & $\begin{array}{l}\text { El tercer molar inferior tiene suficiente espacio } \\
\text { anteroposterior (es decir, por delante del borde anterior de } \\
\text { la rama) para erupcionar. }\end{array}$ & Clased & $\begin{array}{l}\text { El plano oclusal del tercer molar esta al mismo nivel que } \\
\text { el plano oclusal del segundo molar. }\end{array}$ \\
\hline Clase 2 & $\begin{array}{l}\text { Aproximadamente la mitad estí cubierta por la porción } \\
\text { anterior de la rama de la mandibula. }\end{array}$ & Clase B & $\begin{array}{l}\text { El plano oclusal del tercer molar está eatre el plano } \\
\text { oclusal y la linea cervical del segundo molar. }\end{array}$ \\
\hline Clase 3 & $\begin{array}{l}\text { El tercer molar retenido esta totalmente incluido en el } \\
\text { bueso de la rama ascendente. }\end{array}$ & Clase C & $\begin{array}{l}\text { El tercer molar eata bajo la linea cervical del segundo } \\
\text { molar. }\end{array}$ \\
\hline
\end{tabular}




\subsubsection{Clasificación de Winter}

Esta clasificación se basa en el ángulo entre el eje longitudinal del tercer molar y el segundo molar mandibular (Gümrükçü et al., 2021):

- I Posición vertical: $0^{\circ}$ a $10^{\circ}$.

- II Posición mesioangular: $11^{\circ}$ hasta $79^{\circ}$.

- III Posición horizontal: $80^{\circ}$ hasta $100^{\circ}$.

- IV Posición distoangular: $-11^{\circ}$ hasta $-79^{\circ}$.

- V Otro: $111^{\circ} \mathrm{a}-80^{\circ}$ (Incluye dientes mesioinvertidos, distoinvertidos y distohorizontales).

- VI Posición Vestibulolingual.

Figura 2. Representación de diferentes posiciones según Winter.

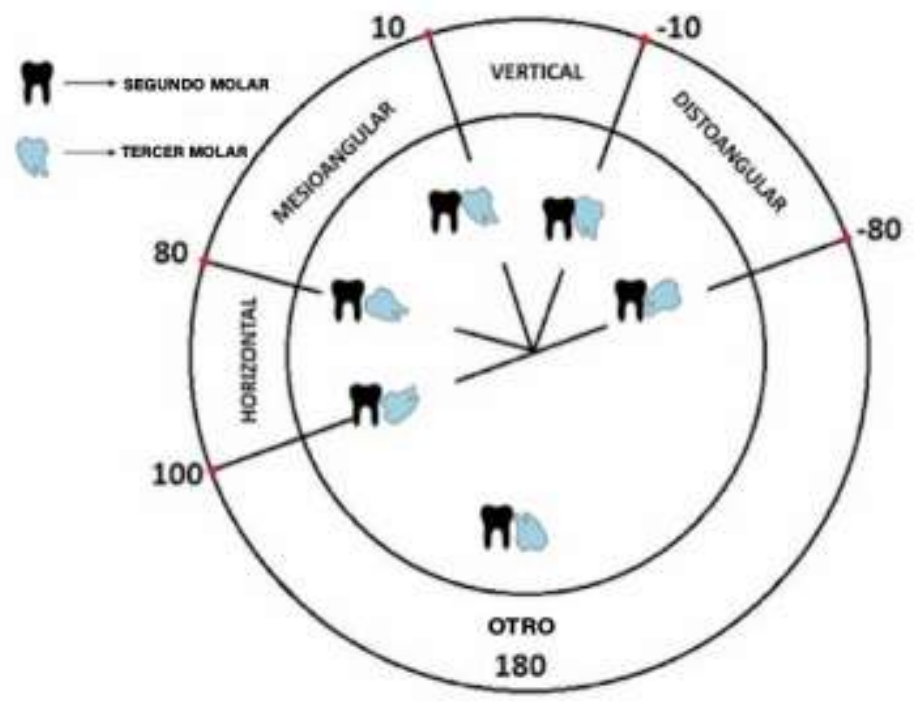

Tomado de: (Gümrükçü et al., 2021).

\subsubsection{Nuevas clasificaciones}

Con la obtención de imágenes tridimensionales se han analizado nuevos métodos de clasificación aplicables para establecer una relación entre los terceros molares y sus estructuras anatómicas adyacentes que revisaremos a continuación.

\subsubsection{Clasificación de Liqun Gu}

Liqun Gu determinó una clasificación para establecer la relación entre la posición del conducto dentario inferior (CDI) con respecto a las raíces del tercer molar inferior (3MM) (Gu et al., 2018).

- Clase I: el conducto dentario inferior se localiza en posición apical. (Figura 3: a, b, c, d)

- Clase II: el conducto dentario inferior se localiza en posición vestibular. (Figura 3: e, f, g, h)

- Clase III: el conducto dentario inferior se ubica en posición lingual. (Figura 3: i, j, k, l)

- Clase IV: el conducto dentario inferior se ubica en posición interradicular. (Figura 3: m, n)

Adicionalmente existen cuatro condiciones que establecen la relación de contacto entre un 3MM y CDI:

1. No existe contacto entre el tercer molar inferior y el conducto dentario inferior. (Figura 3: a, e, i) 
2. Contacto por una línea blanca completa entre el tercer molar inferior y el conducto dentario inferior. (Figura 3: b, $\mathrm{f}, \mathrm{j})$

3. Contacto por medio de una línea blanca defectuosa entre el tercer molar inferior y el conducto dentario inferior. (Figura 3: c, g, k, m)

4. El tercer molar inferior penetra en el conducto dentario inferior. (Fig. 3: d, h, l, n)

La posición vertical del tercer molar mandibular y el conducto dentario se clasifica en dos condiciones según la profundidad de penetración de las raíces:

1. Raíz en la mitad superior del conducto dentario inferior. (Fig. 4: a)

2. Raíz en la mitad inferior del conducto dentario inferior. (Fig. 4: b) (Gu et al., 2018)

Figura 3. Relación entre 3MM y CDI según clasificación de Liqun Gu.
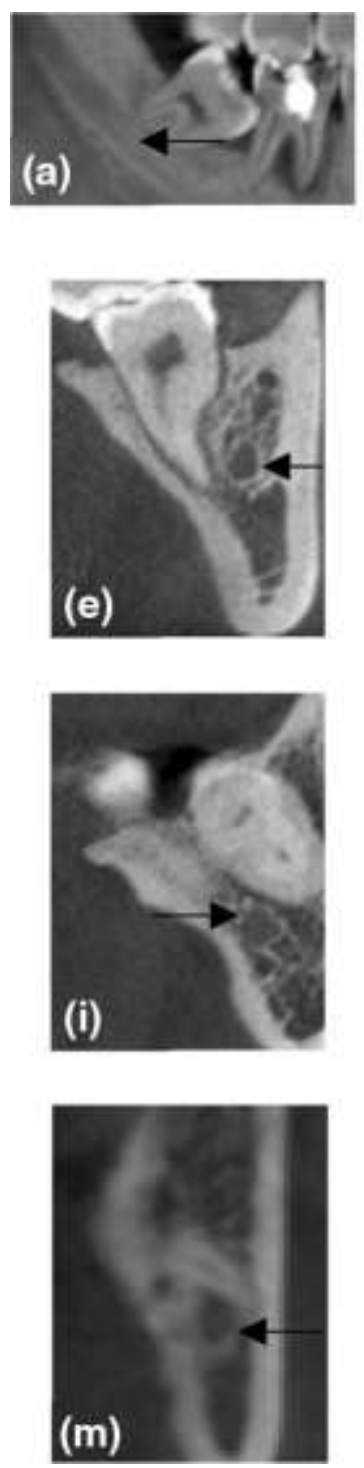
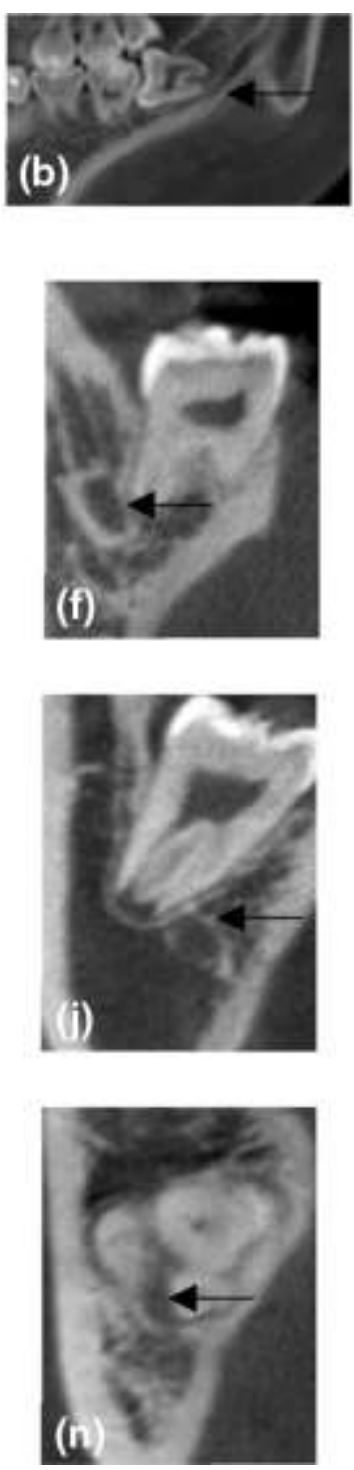
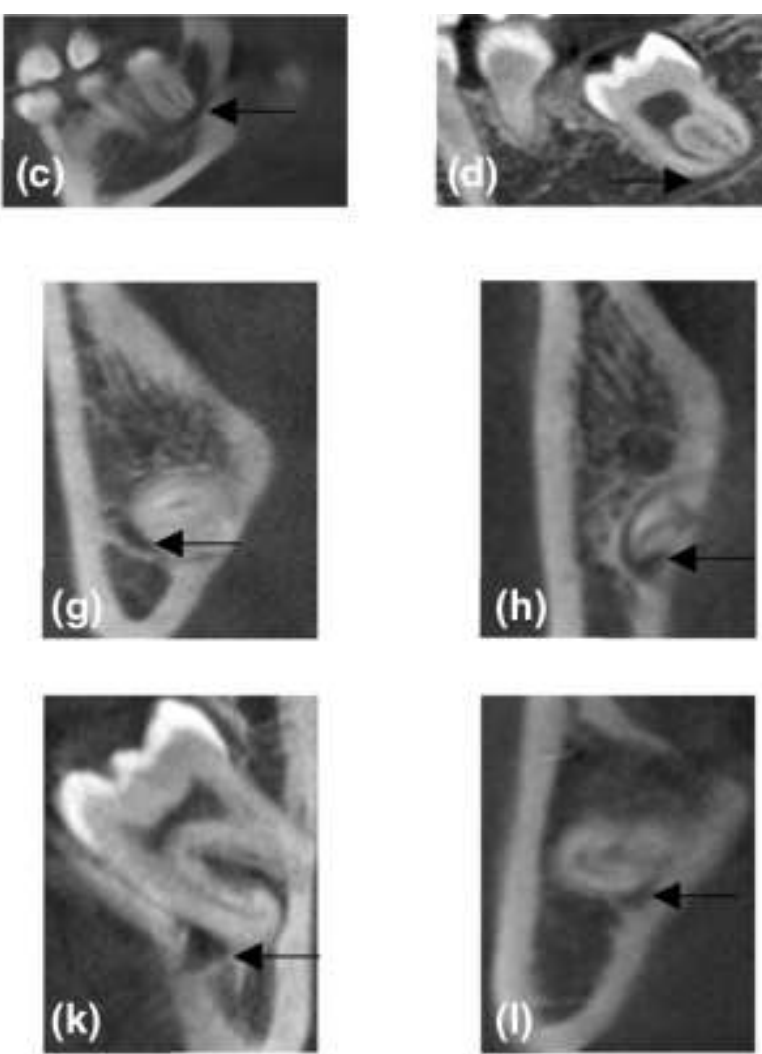

Tomado de: (Gu et al., 2018). 
Figura 4. Profundidad de penetración radicular del 3MM en CDI según Liqun Gu.
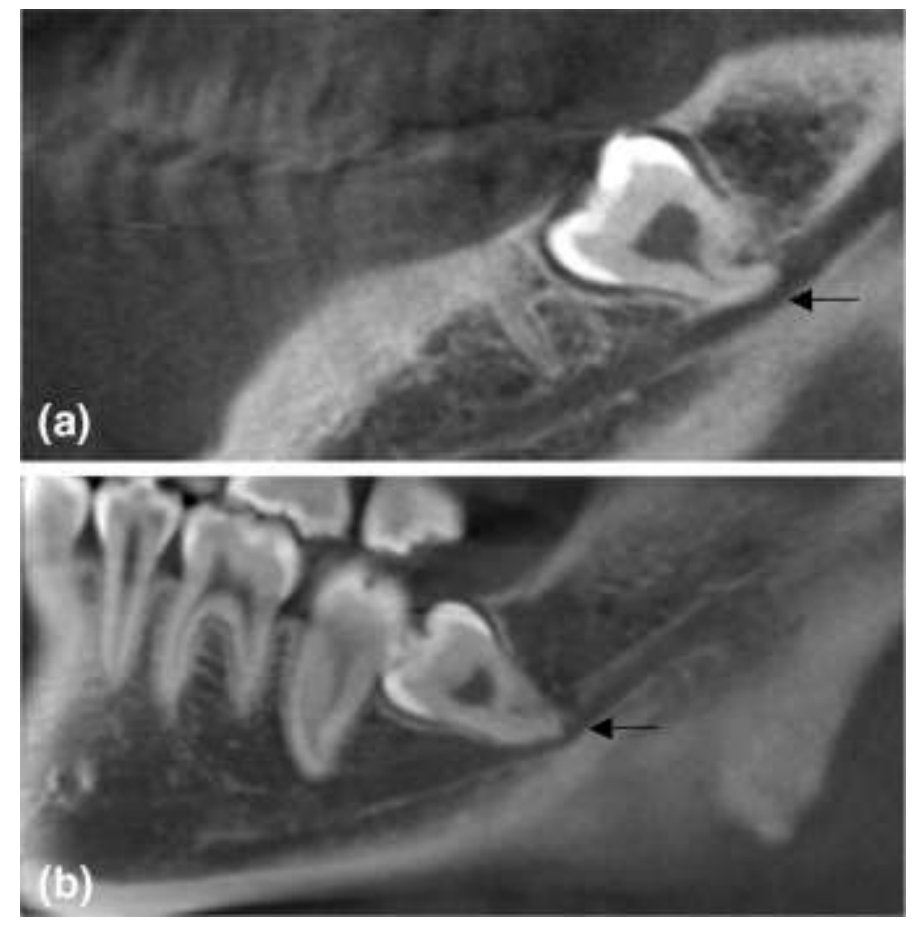

Tomado de: (Gu et al., 2018).

\subsubsection{Clasificación de Ogüz Borahan}

Esta clasificación establece la relación anatómica entre el 3MM y la fosa submandibular (FS), que a su vez aloja en su concavidad a la glándula submandibular (GS) (Aktop et al., 2017).

Según Ogüz Boraham en la CTBC la profundidad de la fosa puede ser evaluada:

- Tipo I: Profundidad de la cavidad $<2 \mathrm{~mm}$.

- Tipo II: Profundidad de la cavidad entre 2 - $3 \mathrm{~mm}$.

- $\quad$ Tipo III: Profundidad de la cavidad $>3 \mathrm{~mm}$.

Figura 5. Clasificación de Ogüz Borahan, trazos para medir la profundidad de la FS.
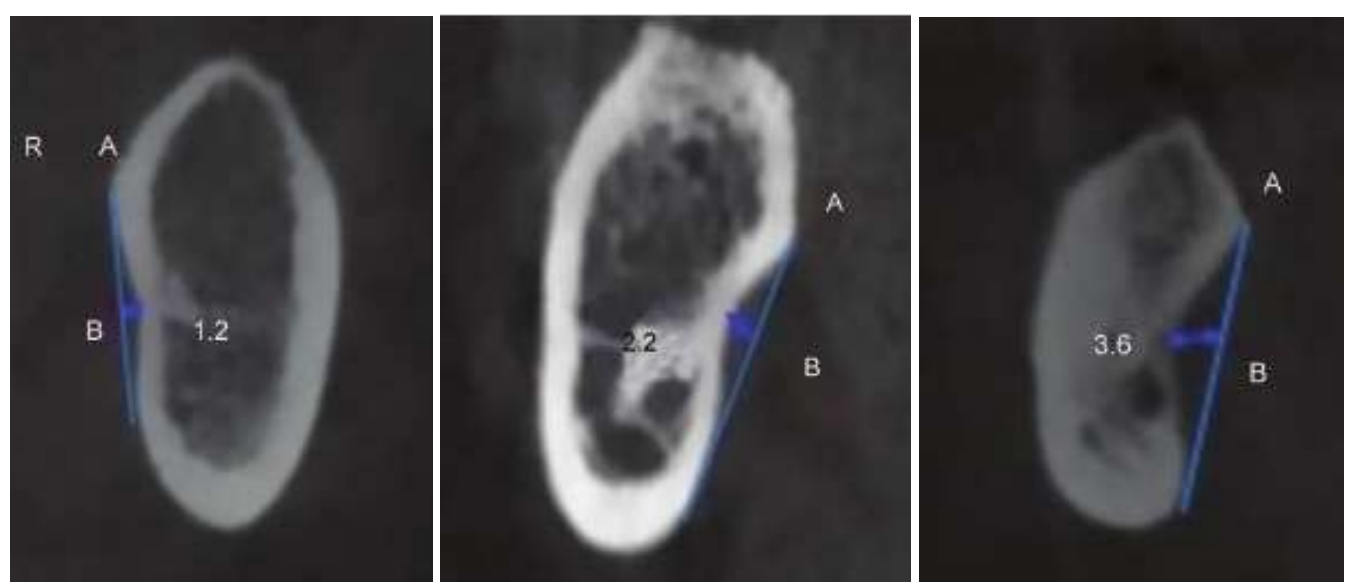

Tomado de: (Mamani Chaiña, 2021) 


\section{Resultados}

3.1 Evaluación de la relación entre los terceros molares mandibulares impactados y el nervio alveolar inferior con tomografía computarizada tridimensional dentaria

Nakayama y Cols., analizan mediante CBCT la posición del 3MM impactado y la relación anatómica con el CDI. Una imagen 3D ofrece la ventaja de visualizar de manera precisa la cercanía entre tejidos dentales y otras estructuras anatómicas; que a diferencia de la RP al ser bidimensional (2D) sobreestima el riesgo de lesión del nervio dentario inferior (NDI) (Nakayama et al., 2009).

Al momento de una extracción de 3MM existe un alto riesgo de lesión al NDI cuando se establece la relación únicamente en una imagen 2D con superposición de estructuras (Nakayama et al., 2009).

3.2 ¿Las imágenes tridimensionales del tercer molar reducen el riesgo de sufrir una lesión del nervio alveolar inferior debido a la extracción?

Los autores Ovejero y Cols., mediante el uso de una RP establecieron que se puede observar en la zona apical del 3MM y el CDI una superposición, el oscurecimiento de la raíz y el estrechamiento de CDl que se pueden correlacionar con ausencia de la cortical del conducto y sugieren un contacto estrecho entre el diente y el nervio. Este contacto directo, se puede observar a mayor detalle a través de en una imagen de CBCT para un análisis en múltiples vistas, pues de evidenciarse estas características se puede asociar con un mayor riesgo de sufrir una lesión entre el 3MM y NDI (Cle-Ovejero et al., 2017).

\subsection{Manejo de terceros molares impactados}

Synan y Stein en su estudio determinaron la importancia de una imagen CBCT para observar de manera minuciosa la relación de las siguientes estructuras (Synan \& Stein, 2020):

- $\quad$ Contacto directo entre el NDI y las raíces del 3MM.

- $\quad$ Ausencia de la cortical del NDI.

- A nivel radicular se aprecia una posición lingual del NDI.

- Posición interradicular del NDI.

- $\quad$ Permite detectar raíces múltiples de 3MM.

\subsection{Relación anatómica de los terceros molares y el canal retromolar}

Una investigación realizada por Rabie y Cols., mediante la utilización del CBCT obtuvieron un análisis del canal retromolar (CR) que se ubica distal al 3MM, este contiene nervios, una arteria y una vena de importancia clínica (Rabie et al., 2019).

La relación entre CR - 3MM se da por el cordón gubernacular, estructura que conecta el diente en formación con las encías, a su vez este se deriva de restos fragmentados de la lámina dental que contiene tejido conectivo, siendo el CR un vestigio del cordón gubernacular. La importancia del CR en este estudio es la fuerte asociación a la malposición dental (posición mesioangular, dientes impactados o rotados) de 3MM (Rabie et al., 2019).

3.5 ¿Indica el sistema de clasificación de Winter o Pell y Gregory la posición apical de los terceros molares mandibulares impactados?

El estudio de Khojastepour y Cols., describieron en RP iniciales una superposición entre los 3MM y el CDI, por lo que decidieron tomar imágenes CBCT para determinar mejor la relación de dichas estructuras (Khojastepour et al., 2019). 
Este estudio reveló que 3MM ubicados hacia lingual presentaban una alta frecuencia en todos los grupos de Winter y Pell y Gregory, además la cortical ósea en esta región se presenta más delgada; mientras que los molares ubicados hacia vestibular con mayor frecuencia presentaban una Clase 3-C; además observaron que los 3MM impactados tenían contacto o invaden el CDI. Los molares mesioangulados tenían un 65.9\% de invasión al CDI (Khojastepour et al., 2019).

Los autores determinaron que, a mayor profundidad de los 3MM, los mismos que se ubicarán más hacia vestibular, y a mayor profundidad de impactación existe mayor posibilidad de lesión del NDI (Khojastepour et al., 2019).

3.6 Fiabilidad de la radiografía panorámica para predecir la proximidad de los terceros molares al canal mandibular: una comparación mediante tomografía computarizada de haz cónico

Otro estudio realizado por Nunes y Cols., en el 2021 analiza la confiabilidad de los estudios radiográficos (RP y CBCT), en el cual se buscó observar la posición de los ápices dentales y su relación con el CDI. Sin embargo, las RP presentan una distorsión referente a las medidas lineales y superposición de estructuras; por lo cual el método radiográfico de mayor precisión es una CBCT debido a los múltiples planos que presenta, además este estudio clasificó de 3 maneras la posición de los 3MM con sus estructuras adyacentes según (Nunes et al., 2021):

- $\quad$ Proximidad entre los 3MM y el CDI.

- Interrupción de la corteza mandibular vestibular o lingual.

- Posicionamiento de los dientes según la clasificación de Pell y Gregory

Este artículo sugiere que la presencia de un área radiográfica con oscurecimiento, desviación o estrechamiento de la raíz, en conjunto con la interrupción del CDI en las RP sugieren la utilización de CBCT (Nunes et al., 2021).

3.7 ¿Son las imágenes panorámicas equivalentes a la tomografía computarizada de haz cónico para clasificar los terceros molares inferiores impactados?

El estudio realizado en Turquía por Danieli Moura Brasil y Cols., entre la concordancia de imágenes RP y CBCT en sus resultados indica, que la distribución en la clasificación de Pell y Gregory fue de 9\% la Clase A, Clase C fue 10.2\% y una prevalencia de la Clase B con el $80.8 \%$ de casos y en la Clase 1 de $7.3 \%$, Clase 2 de $85.3 \%$ y en la Clase 3 de $7.3 \%$ (Brasil et al., 2019).

Además, determinaron que la principal diferencia entre una imagen obtenida por RP subestima el espacio de acomodación de un 3MM, mientras que la imagen por CBCT muestra que el espacio disponible era menor al requerido con una precisión para la evaluación de estos molares (Brasil et al., 2019).

\subsection{El ángulo de inclinación horizontal se asocia con el riesgo de lesión del nervio alveolar inferior durante la extracción} de terceros molares inferiores

Ishii y Cols. en su estudio determinaron la clasificación de Winter y Pell y Gregory por medio de RP, este estudio determinó que la Clase 2 presenta mayor prevalencia con un 55.2\%, seguido de la Clase 3 con un $22.9 \%$ de casos y por último la Clase 1 con un $21.9 \%$ y con respecto a su posición predominaba la Clase B con $68.6 \%$, seguido de la Clase A con $19.0 \%$ y por último la Clase $\mathrm{C}$ con un $12.4 \%$. Según la clasificación de Winter determinaron que la posición mesioangular presenta una mayor prevalencia seguida de una posición horizontal y con frecuencia similar una posición invertida y en menor frecuencia tanto la posición vertical como distoangular (Ishii et al., 2017).

Posteriormente en las imágenes CBCT establecieron que las piezas con Clase 3 presentaban con mayor frecuencia invasión del NDI con respecto a las Clases 1 y 2 (Ishii et al., 2017). 
3.9 Lesión del nervio alveolar inferior: correlación entre los indicadores de riesgo en las radiografías panorámicas y la incidencia de contacto entre los dientes y el canal mandibular en las tomografías computarizadas de haz cónico en una población de Australia Occidental

Winstanley y Cols., en su estudio observaron que el $90 \%$ de casos evaluados presentaban impactación bilateral de los 3MM; por medio de RP establecieron que el $41 \%$ de la muestra presentaba una posición Clase 2 de Pell y Gregory, seguido de la Clase 1 con un $33 \%$ y por último la Clase 3 con un 25\% la misma que en una imagen CBCT demostraba ser propensa a contactar con el NDI, y el 49\% de la muestra mostraba una Clase C de retención. La clasificación de Winter describió que el 36\% tenía una posición mesioangular y el 31\% en sentido horizontal (Winstanley et al., 2018).

La imagen obtenida por medio de CBCT se encargó de evaluar la posición del CDI, así el 34\% de casos se encontraba bajo el 3MM, seguido de un 27\% con posición vestibuloinferior y el 22\% hacia lingual (Winstanley et al., 2018).

3.10 Comparación de los hallazgos de la radiografía panorámica y la tomografía computarizada de haz cónico para la raíz del tercer molar mandibular impactada y la relación del canal del nervio alveolar inferior

Patel y Cols. determinaron la estrecha relación entre la raíz del 3MM impactado, el CDI y la ausencia de cortical entre estas 2 estructuras, mediante RP y CBCT (Patel et al., 2020).

Los datos obtenidos determinan que según Winter a excepción de un 3MM en posición vertical y mesioangular, las demás posiciones se asocian a una ausencia de cortical entre la raíz del 3MM y el CDI. A su vez según Pell y Gregory a excepción de la Clase 1-A, todas las demás clases se asocian a la ausencia de la cortical entre la raíz del 3MM y CDI (Patel et al., 2020).

\subsection{Evaluación de la relación entre el ángulo gonial y los terceros molares mandibulares impactados}

Demirel y Akbulut mediante su investigación demostraron la prevalencia de impactación de los 3MM mediante el uso de RP, cefálica lateral (CL) y CBCT. Se establece una relación entre el molar impactado y el ángulo goniaco (Demirel \& Akbulut, 2020).

En este artículo el ángulo gonial tenía un valor promedio de 121,38 . Según Pell y Gregory la subclasificación 3-C (3MM ubicado por debajo del nivel cervical del 2MM y parcialmente dentro de la rama). Esta investigación mostró que mientras más incluido se encuentra un tercer molar, más abierto será el ángulo gonial (Demirel \& Akbulut, 2020).

3.12 Uso de tomografía computarizada de haz cónico para evaluar hallazgos de imagen significativos relacionados con la impactación del tercer molar mandibular

Según el artículo de Matzen, el mejor método para analizar las estructuras anatómicas cercanas al 3MM es mediante CBCT, obteniendo detalles sobre la angulación y posición de estos, además permite observar la perforación del hueso lingual por las raíces del 3MM (Matzen et al., 2017).

Angulaciones específicas del 3MM son factores de riesgo de patosis relacionada con el segundo molar; estudios previos han demostrado que las posiciones tanto mesioangulada y horizontal se asocian comúnmente con la reabsorción del segundo molar. Además, estas posiciones también se asocian a una pérdida severa de hueso marginal (Matzen et al., 2017). 
3.13 Existe una posible asociación entre los tipos de cara esquelética y la impactación del tercer molar? Un estudio radiográfico retrospectivo

Una investigación de Tassoker y Cols., mencionan la relación entre el biotipo facial y los 3MM que son dientes con alto índice de impactación, lo cual puede surgir debido a la falta de espacio retromolar por insuficiente crecimiento mandibular asociado al biotipo facial (braquifacial, mesofacial y dolicofacial) (Tassoker et al., 2019).

Este artículo analizó la impactación según Winter con una prevalencia de la posición mesioangular en los 3MM. Respecto al biotipo facial, los pacientes braquifaciales demostraron menor prevalencia de impactación del 3M maxilar y mandibular en comparación con dolico y mesofaciales. Evidenciando que pacientes dolicofaciales presentaban una rama ascendente larga combinada con una longitud mandibular corta que predispone a una impactación del 3MM (Tassoker et al., 2019).

3.14 Evaluación de la relación entre la angulación de los terceros molares mandibulares impactados y el grosor del hueso lingual: un estudio clínico prospectivo

Menziletoglu y Cols., determinaron la relación entre la posición de un 3MM y la perforación de la cortical ósea, teniendo en cuenta que la relación era significativa entre la clasificación de Winter y la parte media radicular (Menziletoglu et al., 2019).

Las piezas con dirección distoangular y horizontal presentaban una cortical ósea más delgada. Los molares con angulación vestibulolingual se correlacionan con una perforación a nivel de la cortical lingual y era más común a nivel de la parte media de la raíz, lo cual es únicamente apreciable en una imagen obtenida por medio de CBCT (Menziletoglu et al., 2019).

3.15 Correlación de los marcadores panorámicos de alto riesgo con los hallazgos de la TC de haz cónico en la evaluación preoperatoria de los terceros molares inferiores

Al Ali y Jaber observaron en la CBCT la posición del NDI y la presencia de cortical a nivel del CDI determinando lo siguiente (Al Ali \& Jaber, 2020):

- Presencia o ausencia de cortical ósea entre 3MM y el NDI: determinando que el 27.8\% de casos estudiados presentaron pérdida de la cortical del conducto, por lo que existía un contacto directo entre la raíz y el nervio (Al Ali \& Jaber, 2020).

- Posición del CDI en relación con el 3MM: el 57\% de casos presentaban la ubicación del conducto a nivel vestibular, el $22.8 \%$ de casos hacia lingual, el $17.7 \%$ se ubican inferior al molar mencionado y el $2.5 \%$ a nivel intrarradicular (Al Ali \& Jaber, 2020).

3.16 ¿Tiene impacto el uso de la tomografía computarizada de haz cónico antes de la cirugía del tercer molar mandibular en la planificación del tratamiento?

Baqain y Cols., determinaron que las imágenes por medio de CBCT permiten identificar la relación entre el 3MM y CDI con mayor precisión; en cuanto a proximidad entre el 3MM y el CDI presentaba una prevalencia del $87.1 \%$ a una contraparte en la que el $12.1 \%$ no se encontraban próximos entre sí (Baqain et al., 2020).

La relación vertical entre el 3MM y el CDI establece que un 51.5\% presentaba la pieza coronal al CDI, seguido de un 29.5\% que se encontraban al mismo nivel y el 18.9\% presentaba el molar apical al CDI (Baqain et al., 2020). 
Finalmente determinaron la interrupción de las raíces a nivel del conducto, estableciendo que el 50\% de casos no presentaban interrupción a nivel del conducto; mientras que el 37.9\% de casos presentaban una interrupción entre las raíces de la pieza estudiada y la corteza del CDI (Baqain et al., 2020).

3.17 Asociación entre los terceros molares impactados y la posición del canal mandibular: un análisis morfológico mediante tomografía computarizada de haz cónico

El artículo de Almeida Barros y Cols., analiza la asociación entre la impactación del 3MM con CDI y sus estructuras adyacentes mediante CBCT, para evitar lesiones durante los tratamientos por su estrecha relación (de Almeida Barros et al., 2018).

Según los análisis obtenidos el ángulo de impacto más prevalente de un 3MM fue mesioangular, seguido de la posición vertical y por último horizontal (de Almeida Barros et al., 2018).

En cuanto al CDI y los ápices del 3MM, la posición más prevalente fue bajo las raíces del 3MM, seguido por una localización vestibular. El NDI es más propenso a sufrir una lesión cuando el CDI se ubica en una posición lingual a las raíces de 3MM. La anatomía de las paredes del CDI también se modifica según la proximidad de las raíces de 3MM (de Almeida Barros et al., 2018).

\subsection{La vulnerabilidad de la placa lingual de los terceros molares mandibulares mesioangulares impactados: una medición en imágenes CBCT}

Un estudio realizado por Nguyen y Cols., plantean como objetivo evaluar la cortical lingual del 3MM impactado en posición mesioangular mediante CBCT. Encontraron que la posición más común fue mesioangular; la cortical lingual es la única barrera de tejido duro entre el 3MM impactado y las estructuras anatómicas en la cara lingual de la mandíbula. Se determinó que la cortical lingual era notablemente delgada observándose con mayor frecuencia a nivel del tercio apical de la raíz mesial y en los 3MM de clase A según Pell y Gregory (Nguyen et al., 2021).

\section{Discusión}

El presente estudio nos permite comprender la importancia de una imagen CBCT para obtener una visualización cercana a la realidad de la posición anatómica de un 3MM impactado y sus estructuras adyacentes sin superposición de estructuras (Cle-Ovejero et al., 2017; de Oliveira Moreira et al., 2020; Nakayama et al., 2009; Synan \& Stein, 2020). Una imagen 3D permite observar tres planos anatómicos a diferencia de una imagen 2D que muestra únicamente dos planos de una estructura tridimensional. El profesional debe tener en cuenta que la presencia de oscurecimiento, desviación o estrechamiento de la raíz junto con la interrupción del CDI en una RP determina una nueva toma radiográfica, para la cual el método de elección será un estudio CBCT (Nunes et al., 2021).

$\mathrm{El}$ análisis de los diferentes estudios nos permite observar que las principales clasificaciones aplicadas para determinar la posición de un 3MM son Pell y Gregory y Winter. Tres estudios concuerdan en sus resultados que en Pell y Gregory presenta una prevalencia en cuanto al plano oclusal para la Clase 2, en relación con la cara distal del segundo molar inferior, dos de ellos muestran una prevalencia de Clase B (Brasil et al., 2019; Ishii et al., 2017) a diferencia Winstanley y cols. donde prevalece la Clase C (Winstanley et al., 2018). A su vez Khojastepour y Cols., precisan que la Clase 3-C de Pell y Gregory con posición vestibular y mesioangular invaden el CDI (Khojastepour et al., 2019) dato relevante a nivel clínico.

En cuanto a la clasificación de Winter se concluyó una prevalencia significativa de impactación de 3MM en posición mesioangular entre cinco estudios analizados (de Almeida Barros et al., 2018; Ishii et al., 2017; Nguyen et al., 2021; Tassoker et al., 2019; Winstanley et al., 2018) además, Quirino destaca que la segunda posición más frecuente es vertical y en tercer 
lugar horizontal. Un dato relevante del estudio de Tassoker y Cols., estableció que la posición mesioangular tiene una alta asociación con un biotipo dolicofacial.

La imagen CBCT a diferencia de una RP permite observar el estado de la cortical ósea en relación al 3MM, este dato permitió establecer que los 3MM distoangulados y horizontales presentan una cortical lingual más delgada, además la posición vestibulolingual se relaciona con una perforación de la cortical lingual a nivel medio radicular (Menziletoglu et al., 2019); mientras que los autores Nguyen y Cols., refieren una cortical lingual delgada a nivel del tercio apical de la raíz mesial de un 3MM (Nguyen et al., 2021).

Otra relación que puede ser detectable mediante CBCT es la visualización del CDI - 3MM, en lo cual es posible aplicar la clasificación establecida por Liqun Gu. Un estudio obtuvo por medio de CBCT la posición del CDI con los siguientes resultados: $34 \%$ de casos presentaba el CDI bajo el 3MM, seguido de un 27\% con posición vestibuloinferior del CDI y el 22\% de casos el CDI hacia lingual (Winstanley et al., 2018). Estos resultados se correlacionan con la clasificación de Liqun Gu sobre la posición del CDI y se interpretan con un 34\% como Clase I siendo la predominante, un 27\% de casos Clase II y con un $22 \%$ mostrando menor frecuencia una Clase III.

Liqun Gu describe la posición vertical de un 3MM y el CDI, lo cual es descrito por Zaid y Cols., con los siguientes resultados: un 51.5\% presentaba el 3MM coronal al CDI (Baqain et al., 2020) lo cual indicaría una posición vertical 1; seguido de un $29.5 \%$ que se encontraban al mismo nivel y el $18.9 \%$ presentaba la pieza apical al CDI (Baqain et al., 2020), lo que se interpretaría como una posición 2.

Otra forma de aplicar la clasificación de Liqun Gu de acuerdo con los resultados obtenidos por Quirino sobre el CDI y los ápices del 3MM que muestra una prevalencia del CDI bajo las raíces del 3MM acompañado de una localización vestibular (de Almeida Barros et al., 2018), se podría interpretar como una Clase II-2 según Liqun Gu (posición de CDI respecto a las raíces; posición vertical respecto al CDI) (Gu et al., 2018). Estos resultados sugieren que una posición lingual del CDI con respecto a las raíces del 3MM predispone al NDI a sufrir una lesión durante un procedimiento quirúrgico (de Almeida Barros et al., 2018).

Un artículo incluido en esta revisión establece la afectación de la cortical del CDI con respecto a las raíces del 3MM, los resultados demuestran que todas las posiciones según Winter a excepción de la vertical y mesioangular, presentan ausencia de cortical del CDI (Patel et al., 2020), lo cual analizado en la clasificación de Liqun Gu en base a la relación de contacto de un 3MM y CDI es relación 4. En contraste a esta información otro estudio determinó la interrupción de las raíces a nivel del conducto, indicando que el 50\% de casos no presentaban interrupción a nivel del conducto (Baqain et al., 2020) estableciendo una relación 1; mientras que el 37.9\% presentan interrupción entre las raíces del 3MM estudiado y la corteza del CDI (Baqain et al., 2020) lo cual se interpreta como una relación 3.

La revisión realizada por Shaima y Mohamed según sus resultados que indican un $27.8 \%$ de contacto directo entre la raíz y el nervio (Al Ali \& Jaber, 2020), que según Liqun Gu es una relación 4 de prevalencia; mientras que un 57\% de casos ubican el CDI hacia vestibular (Al Ali \& Jaber, 2020), lo cual determina una Clase II; en contraste con un $22.8 \%$ de casos que muestran el CDI hacia lingual (Al Ali \& Jaber, 2020), otorgando una Clase III. Mientras que un porcentaje representativo del 17.7\% ubica el CDI bajo el 3MM (Al Ali \& Jaber, 2020) atribuyendo una Clase I y con una menor proporción de 2.5\% el CDI está a nivel intrarradicular (Al Ali \& Jaber, 2020) lo que significa una Clase IV.

\section{Conclusiones}

Mediante el análisis de los diferentes estudios incluidos en este meta-análisis podemos concluir que el uso del CBCT nos permite observar de manera detallada la posición y estructuras alrededor de un 3MM. Existe un alto índice de prevalencia 
según Winter del 3MM en posición mesionangular, además destacamos que la ubicación lingual de un 3MM impactado se asocia a la perforación de la cortical lingual visible únicamente en una imagen 3D.

En cuanto a la clasificación de Pell y Gregory la posición de impactación más frecuente es la Clase 2-B. La presente investigación describe nuevas clasificaciones aplicables en una imagen 3D que permiten relacionar al 3MM con sus estructuras adyacentes como lo son las clasificaciones de Liqun Gu y Ogüz Boraham, que recomendamos aplicar en futuras investigaciones para obtener una perspectiva más certera a la posición - relación cercana a la situación clínica de cada paciente.

\section{Referencias}

Aktop, S., Atalı, O., Borahan, O., Gocmen, G., \& Garip, H. (2017). Analyses of anatomical relationship between mandibular third molar roots and variations in lingual undercut of mandible using cone-beam computed tomography. Journal of dental sciences, 12(3), $261-267$.

Al Ali, S., \& Jaber, M. (2020). Correlation of panoramic high-risk markers with the cone beam CT findings in the preoperative assessment of the mandibular third molars. Journal of dental sciences, 15(1), 75-83.

Baqain, Z. H., AlHadidi, A., AbuKaraky, A., \& Khader, Y. (2020). Does the use of cone-beam computed tomography before mandibular third molar surgery Impact treatment Planning? Journal of oral and maxillofacial surgery, 78(7), 1071-1077.

Brasil, D. M., Nascimento, E. H., Gaêta-Araujo, H., Oliveira-Santos, C., \& de Almeida, S. M. (2019). Is panoramic imaging equivalent to cone-beam computed tomography for classifying impacted lower third molars? Journal of oral and maxillofacial surgery, 77(10), $1968-1974$.

Cle-Ovejero, A., Sanchez-Torres, A., Camps-Font, O., Gay-Escoda, C., Figueiredo, R., \& Valmaseda-Castellon, E. (2017). Does 3-dimensional imaging of the third molar reduce the risk of experiencing inferior alveolar nerve injury owing to extraction?: A meta-analysis. The Journal of the American Dental Association, 148(8), 575-583.

de Almeida Barros, R. Q., de Melo, N. B., de Macedo Bernardino, Í, Arruda, M. A. L. L. A., \& Bento, P. M. (2018). Association between impacted third molars and position of the mandibular canal: a morphological analysis using cone-beam computed tomography. British Journal of Oral and Maxillofacial Surgery, 56(10), 952-955.

de Oliveira Moreira, P. E., Normando, D., Pinheiro, L. R., \& Brandão, G. A. M. (2020). Prognosis for the impacted lower third molars: Panoramic reconstruction versus tomographic images. Oral Surgery, Oral Medicine, Oral Pathology and Oral Radiology, $130(6), 625-631$.

Demirel, O., \& Akbulut, A. (2020). Evaluation of the relationship between gonial angle and impacted mandibular third molar teeth. Anatomical science international, 95(1), 134-142.

Giovacchini, F., Paradiso, D., Bensi, C., Belli, S., Lomurno, G., \& Tullio, A. (2018). Association between third molar and mandibular angle fracture: A systematic review and meta-analysis. Journal of Cranio-Maxillofacial Surgery, 46(4), 558-565.

Gu, L., Zhu, C., Chen, K., Liu, X., \& Tang, Z. (2018). Anatomic study of the position of the mandibular canal and corresponding mandibular third molar on cone-beam computed tomography images. Surgical and Radiologic Anatomy, 40(6), 609-614.

Gümrükçü, Z., Balaban, E., \& Karabağ, M. (2021). Is there a relationship between third-molar impaction types and the dimensional/angular measurement values of posterior mandible according to Pell \& Gregory/Winter Classification? Oral radiology, 37(1), 29-35.

Ishii, S., Abe, S., Moro, A., Yokomizo, N., \& Kobayashi, Y. (2017). The horizontal inclination angle is associated with the risk of inferior alveolar nerve injury during the extraction of mandibular third molars. International journal of oral and maxillofacial surgery, 46(12), 1626-1634.

Khojastepour, L., Khaghaninejad, M. S., Hasanshahi, R., Forghani, M., \& Ahrari, F. (2019). Does the Winter or Pell and Gregory classification system indicate the apical position of impacted mandibular third molars? Journal of oral and maxillofacial surgery, 77(11), 2222. e2221-2222. e2229.

Mamani Chaiña, P. V. (2021). Relación de la posición de las terceras molares inferiores con sus estructuras circundantes mediante tomografía Cone Beam en pacientes de 17 a 25 años, Puno 2019-2020

Matzen, L. H., Schropp, L., Spin-Neto, R., \& Wenzel, A. (2017). Use of cone beam computed tomography to assess significant imaging findings related to mandibular third molar impaction. Oral Surgery, Oral Medicine, Oral Pathology and Oral Radiology, 124(5), 506-516.

Menziletoglu, D., Tassoker, M., Kubilay-Isik, B., \& Esen, A. (2019). The assesment of relationship between the angulation of impacted mandibular third molar teeth and the thickness of lingual bone: A prospective clinical study. Medicina oral, patologia oral y cirugia bucal, 24(1), e130.

Nakayama, K., Nonoyama, M., Takaki, Y., Kagawa, T., Yuasa, K., Izumi, K., Ozeki, S., \& Ikebe, T. (2009). Assessment of the relationship between impacted mandibular third molars and inferior alveolar nerve with dental 3-dimensional computed tomography. Journal of oral and maxillofacial surgery, 67(12), 25872591 .

Nguyen, D. A., Le, S. H., Nguyen, C. T. K., Dien, V. H. A., \& Nguyen, L. B. (2021). The vulnerability of lingual plate of the mesioangular impacted mandibular third molars: a measurement on CBCT images. Oral Surgery, 14(2), 106-112. 
Research, Society and Development, v. 10, n.11, e226101119723, 2021

(CC BY 4.0) | ISSN 2525-3409 | DOI: http://dx.doi.org/10.33448/rsd-v10i11.19723

Nunes, W. J. P., Vieira, A. L., de Abreu Guimarães, L. D., de Alcântara, C. E. P., Verner, F. S., \& de Carvalho, M. F. (2021). Reliability of panoramic radiography in predicting proximity of third molars to the mandibular canal: A comparison using cone-beam computed tomography. Imaging Science in Dentistry, 5l(1), 9 .

Patel, P. S., Shah, J. S., Dudhia, B. B., Butala, P. B., Jani, Y. V., \& Macwan, R. S. (2020). Comparison of panoramic radiograph and cone beam computed tomography findings for impacted mandibular third molar root and inferior alveolar nerve canal relation. Indian Journal of Dental Research, 31 (1), 91.

Rabie, C. M., Vranckx, M., Rusque, M., Deambrosi, C., Ockerman, A., Politis, C., \& Jacobs, R. (2019). Anatomical relation of third molars and the retromolar canal. British Journal of Oral and Maxillofacial Surgery, 57(8), 765-770.

Rivera-Herrera, R. S., Esparza-Villalpando, V., Bermeo-Escalona, J. R., Martínez-Rider, R., \& Pozos-Guillén, A. (2020). Análisis de concordancia de tres clasificaciones de terceros molares mandibulares retenidos. Gaceta médica de México, 156(1), 22-26.

Synan, W., \& Stein, K. (2020). Management of Impacted Third Molars. Oral and Maxillofacial Surgery Clinics, 32(4), 519-559.

Tassoker, M., Kok, H., \& Sener, S. (2019). Is there a possible association between skeletal face types and third molar impaction? A retrospective radiographic study. Medical Principles and Practice, 28(1), 70-74.

Winstanley, K. L., Otway, L. M., Thompson, L., Brook, Z. H., King, N., Koong, B., \& O'Halloran, M. (2018). Inferior alveolar nerve injury: Correlation between indicators of risk on panoramic radiographs and the incidence of tooth and mandibular canal contact on cone-beam computed tomography scans in a Western Australian population. Journal of investigative and clinical dentistry, 9(3), e12323. 